\title{
Characterization of Residual Stresses and Retained Austenite on 416 Stainless Steel via X-Ray Diffraction Techniques
}

\author{
Thomas Simmons ${ }^{1, a}$, Gabriel Grodzicki ${ }^{1, b}$, James Pineault ${ }^{2, \mathrm{c}}$, \\ Jeffrey Taptich ${ }^{1, \mathrm{~d}}$, Mohammed Belassel ${ }^{2, \mathrm{e},{ }^{,},}$, Jeffrey Nantais ${ }^{2, \mathrm{f}}$, \\ Michael Brauss ${ }^{1,9}$ \\ ${ }^{1}$ Proto Manufacturing Inc., Taylor, Michigan, USA \\ ${ }^{2}$ Proto Manufacturing Ltd., Ontario, Canada \\ a tsimmons@protoxrd.com, b ggrodzicki@protoxrd.com, ${ }^{c}$ jpineault@protoxrd.com, \\ d jtaptich@protoxrd.com, ${ }^{\mathrm{e}}$ mbelassel@protoxrd.com, ${ }^{\mathrm{f}}$ jnantais@protoxrd.com, \\ gmbrauss@protoxrd.com
}

Keywords: Residual Stress, Retained Austenite, X-Ray Diffraction, Mapping, Stainless Steel

\begin{abstract}
Residual stress (RS) and retained austenite (RA) measurements performed using x-ray diffraction (XRD) techniques have been useful to assist in evaluating the quality of parts both in the field and in production environments for decades. Single spot checks at critical locations are often performed providing basic information for a given testing requirement at a specific location. Although this is useful information, the highest levels of RS or RA present in the component may be missed when performing single spot checks only if surface RS or RA gradients are present. To achieve a more in depth understanding of the range and distribution of RS and RA in a component, a relatively large area was characterized using the mapping approach on a heated and quenched 416 stainless steel sample. The results found using this characterization technique indicate that RS and RA levels are not uniform over the area examined; thus it has been demonstrated that the one point approach is not adequate in this instance since the lowest and highest levels of RS and RA would be missed without mapping. For this reason, it is recommended that the mapping approach be adopted whenever gradients are present to fully and efficiently characterize RS and RA and subsequently to help correctly assess the quality of the part.
\end{abstract}

\section{Introduction}

RS measurements using XRD techniques have been widely applied in many industries, including automotive, aerospace, power generation, medical, and others, on components that are susceptible to stress related failure. The monitoring of RS makes it possible to remove certain components from service before they suffer from cracking or other failure modes, while extending the service life of others. Typically, RS measurements are used as a spot check at failure critical locations often selected using either modeling methods or experience. When the accuracy of models are suspect, or when no experience is available for a specific application, it is possible that areas with RS that negatively impact component life cannot be easily anticipated, predicted, selected, or found. In such instances, areas with potentially harmful stresses can be characterized using RS mapping techniques. Performing a RS map over a large area can produce more useful data from failure critical regions, which can be used to make more cost effective production or component disposition decisions. 
The measurement of RA using XRD has also seen widespread use in quality control and quality assurance. Depending on the specific application, the presence of austenite in the steel can have either positive or negative consequences in terms of component performance and service life. For instance, the presence of RA can improve the fatigue life of bearings and gears composed of high carbon steel, as well as impact fatigue strength in bending or other more complex loading scenarios. The presence of RA may also reduce the load carrying capacity of martensitic/austenitic structures, lower the hardness and resistance to scuffing and indentation, and increase susceptibility to grinder burn and heat checking while grinding [1].

The XRD technique for mapping critical areas can be used when characterizing either RS, RA, or both. The full width at half maximum (FWHM) of the diffraction peaks can also be extracted from the RS and RA map data so that other properties such as the coherent domain size and dislocation density can be analyzed as they may also vary within the mapped region. The XRD technique may be used to collect data at a very specific location over a small region (e.g. a few square millimeters) or a very large area (e.g. several square meters) with individual measurement spot resolution ranging from $0.2 \mathrm{~mm}$ diameter spots up to $2 \mathrm{~mm} \times 5 \mathrm{~mm}$ rectangular spots. As such, detailed RS, RA and/or FWHM maps can be generated without averaging results or assuming microstructural homogeneity over large areas, while capturing the inherent gradients present in failure critical regions on critical components.

\section{Stress Measurement Using the X-Ray Diffraction Technique}

The x-ray diffraction technique can only be applied to crystalline, polycrystalline and semi-crystalline materials [2]. This technique uses the distance between crystallographic planes, i.e. d-spacing, as a strain gage. When the material is in tension the d-spacing increases and when the material is in compression the d-spacing decreases. The presence of residual stresses in the material produces a shift in the x-ray diffraction peak's angular position that is directly measured by a detector [3]. For a known $\mathrm{x}$-ray wavelength $\lambda$ and $\mathrm{n}$ equal to unity, the diffraction angle $2 \theta$ is measured experimentally and the d-spacing (d) is then calculated

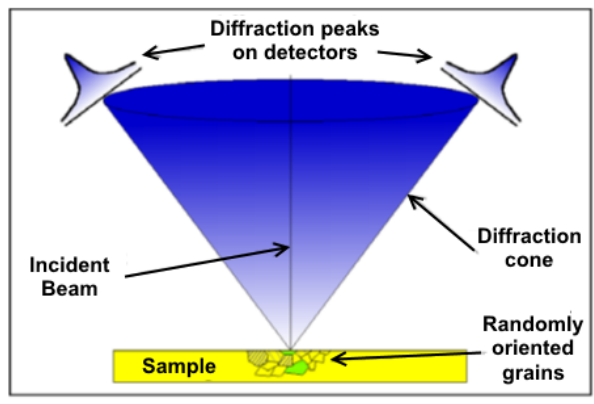

Fig. 1: X-ray diffraction configuration using 2 detectors for stress measurement. The solid angle of the diffraction cone (Debye ring) is determined by the Bragg angle of the diffraction plane. using Bragg's law [4,5]:

$$
n \lambda=2 d \sin \theta
$$

Once the d-spacing is measured for unstressed $\left(d_{0}\right)$ and stressed $(d)$ conditions, the strain is calculated using the following relationship:

$$
\varepsilon=\left(d-d_{0}\right) / d_{0}
$$

For the $\sin ^{2} \psi$ method where a number of d-spacings are measured, stresses are calculated from an equation derived from Hooke's law for isotropic, homogeneous, fine grain materials [1]:

$$
\varepsilon_{\Phi \Psi}=\frac{1}{2} S_{2}\left(\sigma_{\Phi}-\sigma_{33}\right) \sin ^{2} \Psi+\frac{1}{2} S_{2} \sigma_{33}-S_{1}\left(\sigma_{11}+\sigma_{22}+\sigma_{33}\right)+\frac{1}{2} S_{2} \tau_{\Phi} \sin 2 \Psi
$$

where: 
$1 / 2 S_{2}$ and $S_{1}$ are the $x$-ray elastic parameters of the material, $\sigma_{\Phi}$ is the stress in the direction of the measurement, $\psi$ is the angle subtended by the bisector of the incident and diffracted beam and the surface normal, and $\varepsilon \Phi$ is the strain at a given $\psi$ tilt.

Two types of stresses (normal stress $\sigma \Phi$ and shear stress $\tau_{\Phi}$ ) can be calculated from RS measurements performed in a given direction $\phi$ as can be seen in Eq. (3). When the d-spacing vs. $\sin ^{2} \psi$ data are plotted, they have an elliptical form. If a shear stress is not

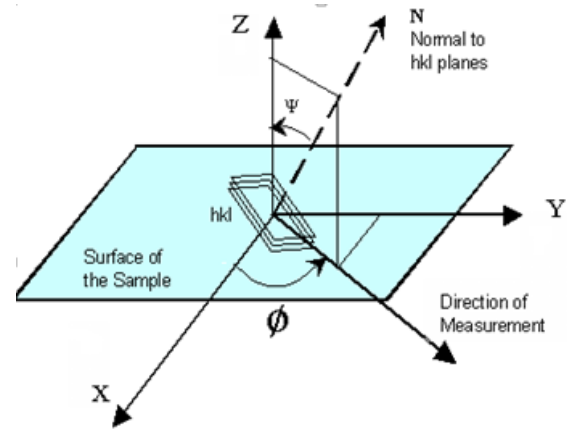

Fig. 2: Definition of the axis and the direction of measurement. present, the ellipse closes and becomes a straight line. If no normal stress is present the slope of the ellipse or line is zero. The opening and slope of the ellipse or line is also dependent upon the goniometer geometry selected, i.e. if the goniometer is working in omega or psi mode or in another non-standard mode such as modified psi or grazing incidence.

\section{Measurement of Percent Retained Austenite}

A unique XRD peak pattern/fingerprint is produced by each constituent phase present in the steel under interrogation (e.g. martensite, austenite, carbides etc.). In samples with a near random grain orientation distribution, the diffracted intensity of the peaks composing each pattern will be proportional to the volume fraction of its corresponding phase [6]

$$
I_{\alpha}^{h k l}=K R_{\alpha}^{h k l} V_{\alpha} / 2 \mu
$$

where:

$K$ is a constant which is dependent upon the selection of instrumentation geometry and radiation, but independent of the material. The $R_{\alpha}^{h k l}$ values are dependent upon the interplanar spacing, the Bragg angle $\theta$, the diffraction $\{\mathrm{hkl}\}$ plane, the crystal structure, and the composition of the phase being measured. Each $R_{\alpha}^{h k l}$ can be calculated from basic principles. $V_{\alpha}$ is the volume fraction of the $\alpha$ phase, and $\mu$ is the linear mass absorption coefficient for the steel.

For steel containing only martensite $(\alpha)$ and austenite $(\gamma)$ and no carbides, we can write for any pair of martensite or austenite $h k l$ peaks:

$$
\frac{I_{\alpha}^{h k l}}{I_{\gamma}^{h k l}}=\left[\left(\frac{R_{\alpha}^{h k l}}{R_{\gamma}^{h k l}}\right)\left(\frac{V_{\alpha}}{V_{\gamma}}\right)\right]
$$

The ratio in Eq. 5 holds if ferrite or martensite and austenite are the only two materials present in the steel. Therefore:

$$
V_{\alpha}+V_{\gamma}=1
$$

Therefore, the volume fraction of austenite $\left(V_{\gamma}\right)$ for the ratio of measured intensities of the $\alpha$ and $\gamma$ peaks to its associated R-value is:

$$
V_{\gamma}=\frac{\frac{I_{\gamma}}{R_{\gamma}}}{\frac{I \alpha}{R_{\alpha}}+\frac{I_{\gamma}}{R_{\gamma}}}
$$


Where more than one martensite and/or austenite peak is collected, the individual ratios of measured intensity to R-value can be summed:

$$
V_{\gamma}=\left(\frac{1}{q} \sum_{j=1}^{q} \frac{I \gamma j}{R \gamma j}\right) /\left[\left(\frac{1}{P} \sum_{i=1}^{1} \frac{I \alpha i}{R \alpha i}\right)+\left(\frac{1}{q} \sum_{j=1}^{q} \frac{I \gamma j}{R \gamma j}\right)\right]
$$

\section{Experimental Procedure}

In the following example a 416 stainless steel (416SS) sample was heated and oil quenched rapidly, allowing austenite to form. A total of sixty RS and RA measurements were performed utilizing a 5 point by 12 point grid encompassing a region of $12 \mathrm{~mm}$ in the transverse direction and $22 \mathrm{~mm}$ in the longitudinal direction (see Fig. 3) using a 1 x $3 \mathrm{~mm}$ aperture. These maps were collected at the surface, at $0.05 \mathrm{~mm}$ below surface, and at $0.254 \mathrm{~mm}$ below surface. The RS was measured in the longitudinal direction. The measurements were performed using a Proto LXRD instrument. The stress measurement parameters are as follows: Cr anode $(\lambda=2.291 \AA)$, hkl (211), Bragg angle $2 \theta=156.4^{\circ}, 1 / 2 \mathrm{~S} 2=5.67 .10^{-6} \mathrm{MPa}^{-1}$. For the RA measurements, diffraction peaks at $2 \theta$ angles of $156^{\circ}, 128^{\circ}, 106^{\circ}$, and $79^{\circ}$ were used corresponding to the $211 \alpha$, $220 \gamma, 200 \alpha, 200 \gamma \mathrm{hkl}$ planes respectively.

\section{Results and Discussion}

The RA maps collected on the 416SS sample were plotted for each of the three depths (see Fig. 4a). It can be seen that the heating and subsequent quenching of the sample produced a localized area with a significant volume fraction of austenite. After electropolishing the sample to a depth of $0.05 \mathrm{~mm}$, the maximum RA found dropped from approximately $30 \%$ at the surface to a maximum of approximately $15 \%$. Once the sample was further electropolished to a depth of $0.254 \mathrm{~mm}$, the maximum volume fraction of austenite in the peak region was found to be less than $5 \%$, comparable to the background level prevalent throughout the sample. These maps indicate that a wide range of RA can be found over a small region in a component and that subsurface measurements are needed to accurately determine the distribution of RA as a function of depth.

Three RS maps were also plotted at the same

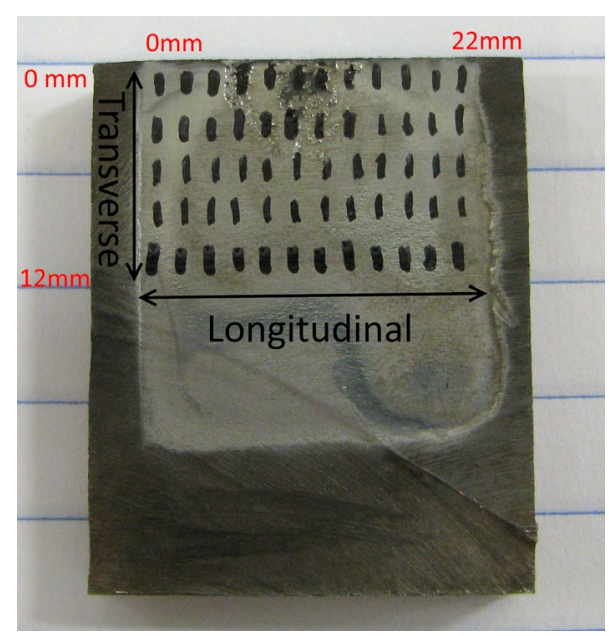

Fig. 3: Location of RS/RA maps on a 416 Stainless Steel Sample at $0.254 \mathrm{~mm}$ below the surface. locations and depths on the same 416SS sample (see Fig. 4b). For the majority of the sampling area mapped on the 416SS sample, the RS was generally compressive. However, near the edges of the mapped region, the RS increased to near neutral, and very locally, into tension. In the center of the mapped region at $0.05 \mathrm{~mm}$ deep, the RS became even more compressive as compared to the surface. These maps indicate that a wide range of RS can be found over a very short distance, even on a largely apparently "uniform" sample. In such cases, it becomes useful to utilize RS mapping to capture any significant changes in RS across a sample. 


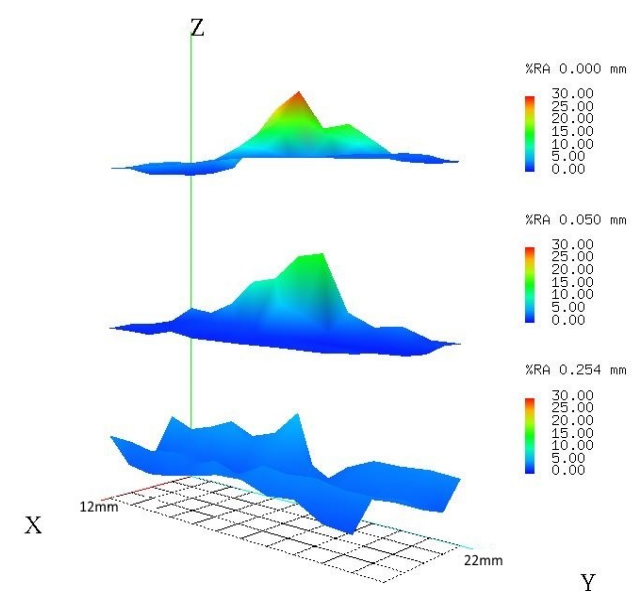

(a)

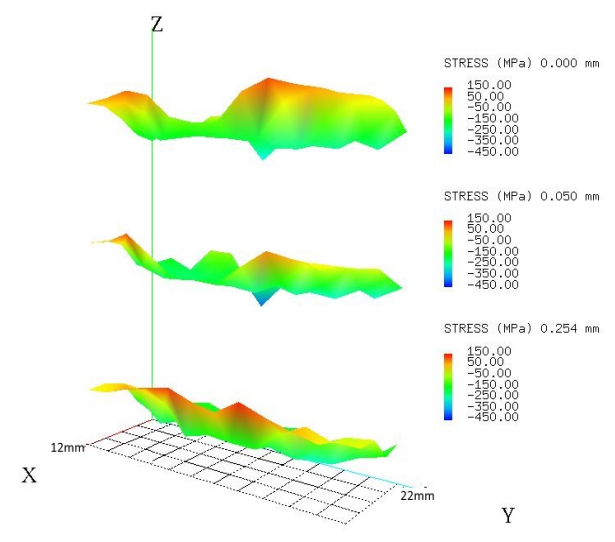

(b)

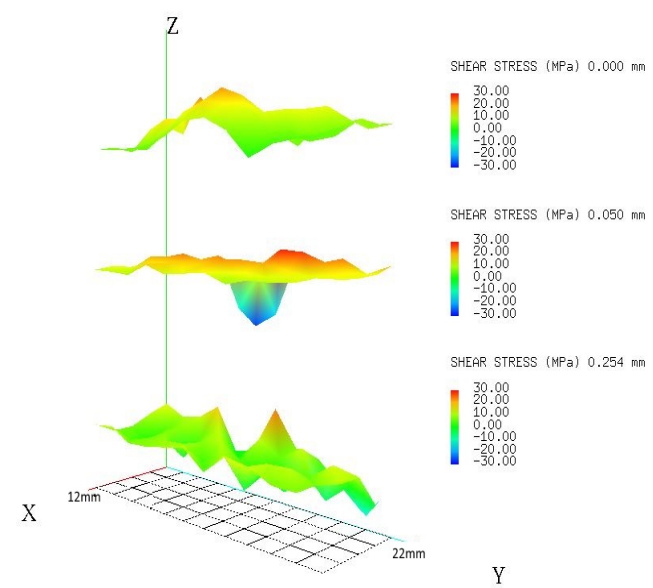

(c)

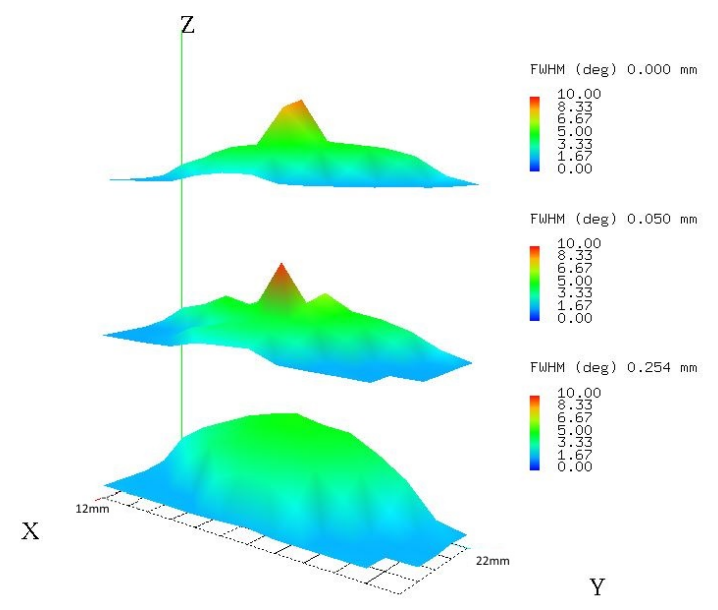

(d)

Fig. 4: (a) RA, (b) RS, (c) shear stress, and (d) FWHM maps on a 416SS sample at surface, 0.05 $\mathrm{mm}$, and $0.254 \mathrm{~mm}$ deep.

The RS maps shown in Fig. 4b also identify the importance of performing RS measurements not only at the surface, but also at depth. A localized region that was slightly more compressive than its surroundings at the surface turned into a region where a large amount of compressive RS was present at a depth of $0.05 \mathrm{~mm}$. Furthermore, the RS in that same region at a depth of 0.254 mm returned to match the RS of its surrounding area. A higher resolution depth profile at that location would provide not only this information, but would also provide a more precise indication of where this area stopped having compressive RS and matched its surroundings. 
These maps also indicate that the neutral/tensile regions of the mapped area were persistent with depth.

The XRD shear stress from the RS maps was also plotted over the same area and depths and varied across the sample area, as well as with depth (see Fig. 4c).

The FWHM of the diffraction peaks can be seen in Fig. 4d. The FWHM of the diffraction peaks (see Fig 4d) varied at the surface with the largest value being at a similar location as the highest value of RA. The highest FWHM wanes with increasing depth into the sample. This is consistent with the fact that the dislocation density increases with hardness [3]. Since FWHM is proportional to dislocation density, these maps thus identify regions of localized hardening at the surface and at depth, and correlate with the associated phase changes observed in the RA maps for this sample.

\section{Conclusions}

The range of RS and RA found in the 416SS sample indicate that mapping is a useful technique for characterizing RS and RA over a large area. Collecting RS and RA maps at the surface and at depth provides a more comprehensive understanding of the surface and subsurface condition of a component. In the case of RA maps, subsurface measurements provide more accurate RA characterization without having the results impacted by surface conditions. Mapping the FWHM of a sample allows an operator to identify localized regions where hardening has occurred, either by heat treatment or by cold working. If RS or RA mapping is not performed, care and experience should be used to select appropriate measurement locations because, as can be seen in the 416SS sample maps, both RA and RS may change significantly over a distance of a few millimeters. Observing and understanding RA, RS, and FWHM maps can provide more meaningful data, which can ultimately be used to improve quality and reliability of critical components.

\section{References}

[1] C. F. Jatczak et. al, "Retained Austenite and its Measurements by X-Ray Diffraction”, SAE SP-453, SAE, 1980.

[2] I. C. Noyan and J. B. Cohen, "Residual Stress - Measurement by Diffraction and Interpretation", Springer-Verlag, New York, 1987.

[3] B. D. Cullity, “Elements of X-ray Diffraction”, Second Edition, Addison-Wesley, Reading Massachusetts, 1978.

[4] M.E. Hilley et.al, "Residual Stress Measurement by X-ray Diffraction” SAE HS-784, SAE, 2003.

[5] H.P. Klug, L.E. Alexander, "X-ray Diffraction Procedures for Polycrystalline and Amorphous Materials”, Second Edition, Wiley-Interscience, New York, 1974.

[6] ASTM E975-03, "Standard Practice for X-Ray Determination of Retained Austenite in Steel with Near Random Crystallographic Orientation”, 2008. 